\title{
Collagen content in the vastus lateralis and the soleus muscle following a 90-day bed rest period with or without resistance exercises
}

\author{
Rasmus Oestergaard Nielsen ${ }^{1,5}$ \\ Peter Schjerling ${ }^{2}$ \\ Per Tesch ${ }^{3}$ \\ Per Stål 4 \\ Henning Langberg ${ }^{5}$
}

1 Department of Public Health, Section of Sport Science, Aarhus University, Denmark

2 Institute of Sports Medicine, Department of Orthopedic Surgery M, Bispebjerg Hospital and Center for Healthy Aging, Faculty of Health and Medical Sciences, University of Copenhagen, Denmark

3 Department of Physiology and Pharmacology, Karolinska Institutet, Stockholm, Sweden

4 Department of Integrative Medical Biology, laboratory of Muscle Biology, Umeå University, Sweden

5 Copen Rehab, Section for Social Medicine, Department of Public Health, University of Copenhagen, Denmark

Corresponding author:

Henning Langberg

Copen Rehab, Section for Social Medicine,

Department of Public Health, University of Copenhagen

Henrik Pontoppidansvej 4, st

2200 Copenhagen N, Denmark

E-mail: langberg@sund.ku.dk

\section{Summary}

Introduction: spaceflight seems associated with deterioration of the function of the skeletal muscles. Since muscle collagen is critical for muscle function, an improved understanding of the content of the muscle collagen during long-term inactivity seems important. Bed-rest with in-bed resistance training serves as a proxy for the conditions in space. Therefore, ground-based studies may improve the understanding of the consequences of long-term inactivity.

Purpose: the purpose is to compare the change in collagen protein in the vastus lateralis (VL) and the soleus (SOL) muscle amongst persons exposed to a 90 -day bed rest with or without resistance exercise.

Methods: an explorative analysis was completed based on data from a randomized, controlled trial. The intervention group (BRE, SOL $n=4, V L n=8$ ) performed supine-based squat exercises, whereas the controls ( $B E, S O L n=6, V L n=12$ ) remained inactive during follow-up. Muscle biopsies from vastus lateralis and soleus were taken at baseline (pre) and after 90-days' follow-up (post). Muscle collagen ( $\mu \mathrm{g}$ collagen $/ \mathrm{mg}$ protein) was quantified. Two-way repeated measurements ANOVA was used to compare the interaction between the intervention (BRE/BR) and time (pre/post) for each muscle.

Results: the collagen content of VL was similar between pre and post in the BRE group (-3.8 $\mu \mathrm{g}$ collagen $/ \mathrm{mg}$ protein $[95 \% \mathrm{Cl}:-22.0 ; 14.4], \mathrm{p}=0.68$ ) while it rose amongst individuals in the BR group (14.9 $\mu \mathrm{g}$ collagen/mg protein [95\% Cl: $-0.01 ; 29.7$ ], $\mathrm{p}=0.05$ ). The difference of 18.66 [95\% Cl: -6.5 ; 43.9] between BRE and BR across time was, however, not significant $(p=0.14)$. No significant reduction in SOL muscle collagen content was observed from pre to post in the BR group $(-9.3 \mu \mathrm{g}$ collagen/mg protein [95\% $\mathrm{Cl}:-24.9 ; 6.4], \mathrm{p}=0.25)$ or in the BRE group $(-6.5 \mu \mathrm{g}$ collagen $/ \mathrm{mg}$ protein [95\% Cl: $-25.6 ; 12.6], p=0.50)$. There was no difference in the effect of BR versus BRE over time (mean difference $\mathbf{- 2 . 7 8} \boldsymbol{\mu g}$ collagen/mg protein [95\% Cl: $-29.7 ; 24.1], \mathrm{p}=0.82$ ).

Conclusion: muscle collagen content in the VL or SOL muscle does not seem to differ after a 90-day bed rest period with or without squat exercises.

KEY WORDS: exercise, bed rest, spaceflight, collagen, muscle.

\section{Introduction}

Continuous exercise is important to maintain physical performance, bone mass and muscle function. During spaceflight, astronauts are exposed to a gravitational environment, which limits their ability to perform weight-bearing exercises. As a consequence, it is widely accepted that spaceflight is associated with atrophy and deterioration of the function of the skeletal muscles ${ }^{1}$. Although this belief is debatable because supporting data from long-term space missions are lacking ${ }^{2,3}$, studies have been conducted to illuminate the beneficial effects of resistance training during ground-based bed rest $(B R)^{4-6}$. Consequently, evidence surfaced during the past decades that such inflight exercise simulating resistance training, or equivalently bed rest resistance exercise (BRE), may 
counter act the deleterious effect of inactivity on muscle function ${ }^{4-6}$.

Since muscle collagen is critical for muscle function ${ }^{6}$, an improved understanding of the content of the muscle collagen during long-term inactivity seems important. The effect of BRE on the collagen level in the vastus lateralis muscle and the soleus muscle is, still, an important area to study, since the vastus lateralis contributes to shock-absorption at foot strike and the soleus muscle is a main contributor to propulsion during human gait ${ }^{7,8}$. A period of inactivity without resistance exercise may affect walk and run, possibly because the content of muscle collagen, amongst other factors like muscle volume, strength or fatigability, changes.

Interestingly, Haus et al. ${ }^{6}$ reported a significant increase from $3 \pm 1$ to $18 \pm 3 \mu \mathrm{g} / \mathrm{mg}$ muscle wet weight in the soleus muscle, while the collagen concentration in the vastus lateralis remained similar, following a 90-day, bed rest period without resistance exercise. These data were, however, not compared to an intervention group completing resistance exercise. The purpose of the present study was, therefore, to compare the muscle collagen content ( $\mu \mathrm{g}$ collagen $/ \mathrm{mg}$ protein) in the vastus lateralis muscle and in the soleus muscle following a 90-day period amongst ten persons exposed to BR or BRE.

\section{Methods}

The trial meets the ethical standards of the Muscles, Ligaments and Tendons Journal ${ }^{9}$.

\section{Design}

The design was a randomized controlled trial with a 90-day follow-up completed at the Institut de Médecine et de Physiologie Spatiales (MEDES) in Toulouse, France 4,10 . The experimental design has been described elsewhere ${ }^{4,10,11}$. All subjects gave informed consent to participate in the study, which was approved by the local Ethical Committee in Toulouse, France [le Comité consultative de protection des personnes dans la recherche biomedicale (C.C.P.P.R.B.) de Toulouse I].

\section{Subjects}

The bed rest procedure was organised by staff at MEDES. All subjects were placed in a supine position and maintained a 6-degree head-down tilt position at all times during bed rest. During transportation, toilet, shower and training, the subjects were restricted to a head-down recumbent position ${ }^{10}$. Muscle biopsies were obtained from the vastus lateralis (VL), and for comparison, from the soleus (SOL) muscle. The subjects were randomly allocated to one of the following two exposure groups: bed rest without resistance exercise (BR, SOL $n=6, V L n=12$ ) or bed rest with resis- tance exercise (BRE, SOL $n=4, V L n=8)$. The subjects in the BR group were $32 \pm 2$ years, had a length of $174 \pm 2 \mathrm{~cm}$, weight of $70 \pm 2 \mathrm{~kg}$ and a BMl of $23 \pm 1 \mathrm{~kg} \mathrm{~m}^{-2}$. The mean age in the BR group was $32 \pm 2$ years, length was $174 \pm 1 \mathrm{~cm}$, weight was $72 \pm 2 \mathrm{~kg}$ and $\mathrm{BMI}$ was $24 \pm 1 \mathrm{~kg} \mathrm{~m}^{-2}$.

\section{Exposure}

Primary exposure was resistance exercise. The exercise intervention consisted of 29 training sessions during the 90-day follow-up. In each session, twojoint squat exercises consisting of four sets of seven maximal concentric and eccentric repetitions of supine squats were completed in a 6-degree headdown tilt position. In addition to the primary exposure, time was included using two discrete time-points: prior to bed rest (pre) and after 90-days' follow-up (post).

\section{Outcome}

The primary outcome was the paired change in muscle collagen content ( $\mu \mathrm{g}$ collagen/mg protein) between pre to post. Muscle biopsies were taken from the soleus and vastus lateralis prior to and at the end of follow-up. Following each biopsy, a piece of the muscle was embedded in Tissue Tek and immediately frozen in is opentane cooled by liquid nitrogen. The embedded biopsies were then stored at $-80^{\circ} \mathrm{C}$.

\section{Collagen measurement}

8-12 sections of $20 \mu \mathrm{m}$ where cut in a cryostat and hydrolysed in $0.5 \mathrm{ml} 6 \mathrm{M} \mathrm{HCl}$ at $110^{\circ} \mathrm{C}$ as described in Syk et al. ${ }^{12}$. Following evaporation of $\mathrm{HCl}$ in a heating block $\left(95^{\circ} \mathrm{C}\right.$, repeated with $\left.\mathrm{H}_{2} \mathrm{O}\right)$, the hydrolysates were dissolved in acetate-citrate buffer and $150 \mu \mathrm{l}$ used for colorimetric measurement and hydroxyproline by the chloramine-T method ${ }^{13}$. Total amino acid content was measured in the hydrolysate with the OPA-NAC method as previously described $^{14}$, except the reactions which were performed in microtiter plates on $10 \mu \mathrm{l}$ sample in $200 \mu \mathrm{l}$ and measured in a fluorimeter (ex $340 \mathrm{~nm}$, em 450476 nm, Victor3 1420 multi-label counter, PerkinEImer, Waltham, MA, USA). Hydroxyproline and valine were used for standard curves, but bovine collagen (C9879, Sigma) and bovine serum albumin (as total protein) (A3912, Sigma) were included and the final results related to these measurements to yield mg collagen per mg total protein. A histological study of the different conditions might provide better insight of muscle response and result reliability. Such study was completed (picrosirius red). Unfortunately, the collagen distribution was impossible to quantify. Therefore, no histological data is presented. However, pictures of the cross-section area from a biopsy in the soleus muscle and the vastus 
Collagen content in the vastus lateralis and the soleus muscle following a 90-day bed rest period with or without resistance exercises

lateralis muscle prior to- and after the bed rest period from a person in the BR group and a person in the BRE group are visualised in the supplementary material.

\section{Statistical analyses}

In STATA (version SE 13, College Station, Texas, USA), a two-way repeated measurements ANOVA was used to compare the interaction between group (BR/BRE) and time (pre/post). Results are presented according to the recommendations by Knol and Vander Weele ${ }^{15}$. Significance was accepted at $P<0.05$.

\section{Results}

Descriptive data on the collagen content ( $\mu \mathrm{g}$ collagen/mg protein) in the soleus and vastus muscles prior to and after bed rest is presented in Table 1. The collagen content of vastus lateralis was similar between pre and post in the BRE group (-3.8 $\mu \mathrm{g}$ collagen/mg protein $[95 \% \mathrm{Cl}:-22.0 ; 14.4], p=0.68)$ while it rose amongst individuals in the BR group (14.9 $\mu \mathrm{g}$ collagen/mg protein $[95 \% \mathrm{Cl}:-0.01 ; 29.7], \mathrm{p}=0.05)$ as presented in Table 2.The difference of 18.66 [95\% Cl:
-6.5; 43.9] between BRE and BR across time was, however, not significant $(p=0.14)$.

In the soleus muscle, there was no significant change in the BR group $(-9.3 \mu \mathrm{g}$ collagen/mg protein $[95 \% \mathrm{Cl}:-24.9 ; 6.4], \mathrm{p}=0.25)$ nor in the BRE group $(-6.5 \mu \mathrm{g}$ collagen/mg protein [95\% Cl: -25.6 ; 12.6], $p=0.5)$ from pre to post (Tab. 3). There was no difference in the effect of BR versus BRE over time (mean difference $-2.78 \mu \mathrm{g}$ collagen/mg protein [95\% Cl: -29.7; 24.1], $p=0.82)$. In Figure 1, the absolute collagen levels at baseline and at the end of follow-up in the vastus lateralis and soleus muscles are visualised.

\section{Discussion}

In the present study, persons were randomised to bed rest without exercise or bed rest with a 29-session resistance-training regime targeted at the knee extensors. Over a 90-day period, the collagen content of the vastus lateralis seemed to decrease in the BRE group while an increased collagen level was present in the BR group. This interaction between time and intervention groups was, however, not significant $(p=0.14)$. We do not have any reasonable explanation of this result. Possibly, resistance exercise does not

Table 1. Descriptive data on the collagen level ( $\mu$ g Collagen/mg Protein) in the soleus- and vastus muscles at different time-points and across intervention. $\mathrm{Cl}=$ Confidence Interval.

\begin{tabular}{|c|c|c|c|c|}
\hline \multirow[b]{2}{*}{ Muscle } & \multicolumn{2}{|c|}{ Bed rest only } & \multicolumn{2}{|c|}{ Bed rest and exercise } \\
\hline & Time-point & Estimate $[95 \% \mathrm{Cl}]$ & Time-point & Estimate $(95 \% \mathrm{Cl})$ \\
\hline \multirow[t]{2}{*}{ Soleus } & Pre & $64.3[52.6 ; 75.9]$ & Pre & $41.7[32.5 ; 50.9]$ \\
\hline & Post & $55.0[37.0 ; 73.0]$ & Post & $35.2[15.7 ; 54.8]$ \\
\hline \multirow[t]{2}{*}{ Vastus } & Pre & $45.6[33.4 ; 57.8]$ & Pre & $52.4[37.2 ; 67.6]$ \\
\hline & Post & $60.5[47.0 ; 74.0]$ & Post & $48.6[32.9 ; 64.2]$ \\
\hline
\end{tabular}

Table 2. The effect of exercise during bed rest on the collagen content ( $\mu$ g Collagen/mg Protein) in the vastus lateralis muscle. Results are presented as the interaction between time (pre/post) and group (control/intervention). $\mathrm{Cl}=$ Confidence interval. The collagen level in the reference group was: $45.6[33.4 ; 57.8]$.

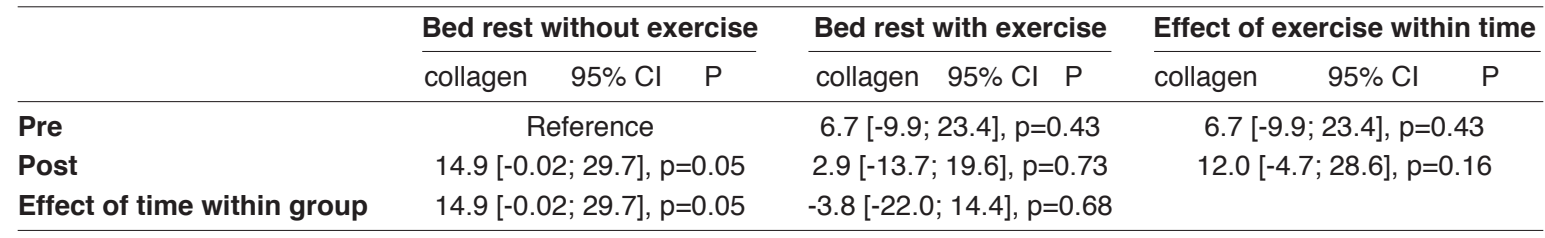

Table 3. The effect of exercise during bed rest on the collagen content ( $\mu \mathrm{g}$ Collagen $/ \mathrm{mg}$ Protein) in the soleus muscle. Results are presented as the interaction between time (pre/post) and group (control/intervention). $\mathrm{Cl}=\mathrm{Confi}-$ dence interval. The collagen level in the reference group was: 64.3 [52.6; 75.9]. The relative excess risk due to interaction on an additive scale $=2.8, P=0.91$.

\begin{tabular}{|c|c|c|c|c|c|c|c|c|c|}
\hline & \multicolumn{3}{|c|}{ Bed rest without exercise } & \multicolumn{3}{|c|}{ Bed rest with exercise } & \multicolumn{3}{|c|}{ Effect of exercise within time } \\
\hline & collagen & $95 \% \mathrm{Cl}$ & $\mathrm{P}$ & collagen & $95 \% \mathrm{Cl}$ & $\mathrm{P}$ & collagen & $95 \% \mathrm{Cl}$ & $\mathrm{P}$ \\
\hline Pre & \multicolumn{3}{|c|}{ Reference } & \multicolumn{3}{|c|}{$-22.6[-41.0 ;-4.2], p=0.02$} & \multicolumn{3}{|c|}{$-22.6[-41.0 ;-4.2], p=0.02$} \\
\hline Post & \multicolumn{3}{|c|}{$-9.3[-24.9 ; 6.4], p=0.25$} & \multicolumn{3}{|c|}{$-29.1[-47.5 ;-10.6], p=0.001$} & \multicolumn{3}{|c|}{$-19.8[-38.2 ;-1.4], p=0.04$} \\
\hline Effect of time within group & \multicolumn{3}{|c|}{$-9.3[-24.9 ; 6.4], p=0.25$} & \multicolumn{3}{|c|}{$-6.5[-25.6 ; 12.6], p=0.5$} & & & \\
\hline
\end{tabular}




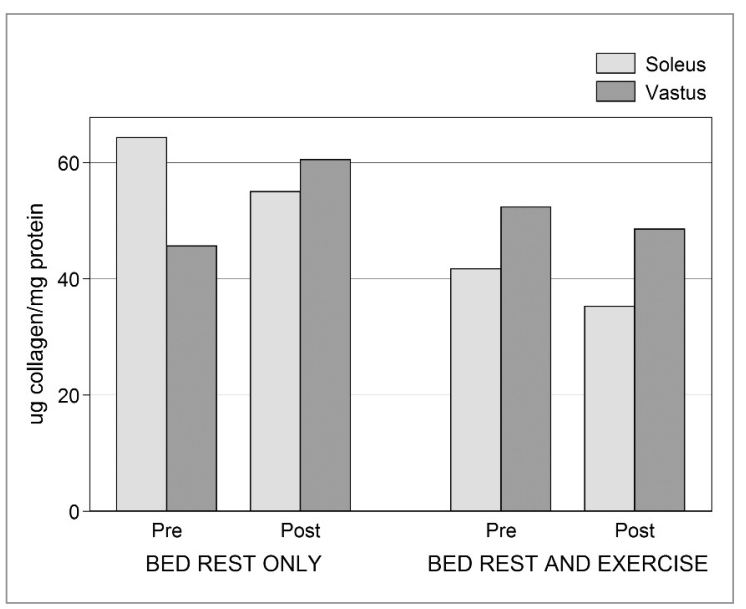

Figure 1. LZW.

diminish the reduction in the collagen in the ankle plantar flexor nor knee extensors indicating that it is important to develop different loading regimes for use in space. Potentially eccentric components of the exercise needs to be improved to generate enough load and possibly micro-fractures in the collagen to stimulate an adaptive healing response leading to maintained content of collagen in the muscles. Importantly, more studies are needed to verify this assumption and investigate the association between other measures like muscle fibre type/size analysis, muscle atrophy, strength or fatigability between those who exercise and their non-exercising peers as done by Haus et al ${ }^{6}$. In the soleus muscle, the change in absolute collagen content was insignificant in BRE and BE (Tab. 3) and no interaction between intervention group and time was observed $(p=0.82)$. Based on this, squat-based exercises do not seem to affect the collagen content in the soleus muscle. It is well-known that squat exercises target the knee extensors. Such exercise may not stress the ankle plantar flexors sufficiently to produce an effect. If exercises targeted at the ankle plantar flexors had been performed, it may have led to significant changes in collagen content between BRE and BR. Another limitation besides the type of exercise is the small sample of ten persons, which likely could have led the primary result to be underpowered. Therefore, future research should address the effect of exercises targeted at the ankle plantar flexors amongst a greater sample.

The strength of the present trial was the 90-days' bed rest period and the study design (randomised, controlled trial). Although confounding may be limited owing to the design, it may also be introduced because of lack of biopsies on all randomised participants. In particular, a limited number of persons had biopsies taken from the soleus. Based on this, bias because of confounding could be introduced on the soleus data. However, there were no significant differences between age, height, weight and BMI between the BRE and BR leaving out these co-variates as confounders. Still, unknown confounders could have biased the result.
In the present study, there was no effect of squatbased resistance exercise on the change in muscle collagen content in the two muscles examined. Importantly, resistance exercise may lead to a maintained muscle volume (prevent atrophy) ${ }^{5,6}$ and lead to preserved bone mineral concentration ${ }^{5}$ during bed rest. Therefore, astronauts should still be encouraged to exercise regularly during spaceflight missions.

\section{Conclusion}

In the present study, resistance training did not affect the collagen content in the vastus lateralis or soleus muscle to a greater or lower level than non-exercising controls. Consequently, there was no effect of squatbased resistance exercise on the change in muscle collagen content in the two muscles.

\section{Acknowledgements}

Camilla Sørensen and Ann-Christina Reiman are acknowledged for excellent technical assistance.

This study was supported by the European Space Agency.

\section{Conflicts of interests}

The Authors declare no conflicts of interest.

\section{Author contributions}

Hypothesis and synthesis: PS, HL.

Data collection: PT.

Data management: PS.

Statistical analysis: RON, TON.

Interpretation: RON, PS, TON

Writing the manuscript: RON, PS, HL

Revising the manuscript for important intellectual content: TON, PS, HL, PT

\section{References}

1. Edgerton VR, Roy RR. Neuromuscular adaptations to actual and simulated spaceflight. Handbook of Physiology: Adaptation to the environment. 1st ed.Oxford University Press, New York. 1995;721-763.

2. LeBlanc A, Lin C, Shackelford L, Sinitsyn V, Evans H, Belichenko $\mathrm{O}$, et al. Muscle volume, MRI relaxation times (T2), and body composition after spaceflight. J Appl Physiol (1985) 2000; 89(6):2158-2164.

3. Lambertz D, Perot C, Kaspranski R, Goubel F. Effects of longterm spaceflight on mechanical properties of muscles in humans. J Appl Physiol (1985) 2001;90(1):179-188.

4. Alkner BA, Tesch PA. Knee extensor and plantar flexor muscle size and function following 90 days of bed rest with or without resistance exercise. Eur J Appl Physiol. 2004;93(3):294305.

5. Rittweger J, Frost HM, Schiess H, Ohshima H, Alkner B, Tesch $\mathrm{P}$, et al. Muscle atrophy and bone loss after 90 days' bed rest 
Collagen content in the vastus lateralis and the soleus muscle following a 90-day bed rest period with or without resistance exercises

and the effects of flywheel resistive exercise and pamidronate: results from the LTBR study. Bone. 2005;36(6):1019-1029.

6. Haus JM, Carrithers JA, Carroll CC, Tesch PA, Trappe TA. Contractile and connective tissue protein content of human skeletal muscle: effects of 35 and 90 days of simulated microgravity and exercise countermeasures. Am J Physiol Regul Integr Comp Physiol. 2007;293(4):R1722-1727.

7. Schache AG, Blanch PD, Dorn TW, Brown NA, Rosemond D, Pandy MG. Effect of running speed on lower limb joint kinetics. Med Sci Sports Exerc. 2011;43(7):1260-1271.

8. Dorn TW, Schache AG, Pandy MG. Muscular strategy shift in human running: dependence of running speed on hip and ankle muscle performance. J Exp Biol. 2012;215(Pt 11):1944-1956.

9. Padulo J, Oliva F, Frizziero A, Maffulli N. Muscles, Ligaments and Tendons Journal. Basic principles and recommendations in clinical and field science research. MLTJ. 2013;4:250-252.

10. Alkner BA, Tesch PA. Efficacy of a gravity-independent resistance exercise device as a countermeasure to muscle atrophy during 29-day bed rest. Acta Physiol Scand. 2004;181(3):345357.

11. Tesch PA, Trieschmann JT, Ekberg A. Hypertrophy of chronically unloaded muscle subjected to resistance exercise. J Appl Physiol (1985). 2004;96(4):1451-1458.

12. Syk I, Agren MS, Adawi D, Jeppsson B. Inhibition of matrix metalloproteinases enhances breaking strength of colonic anastomoses in an experimental model. Br J Surg 2001; 88(2):228-234.

13. Rehn M, Agren MS, Syk I. Collagen levels are normalized after decompression of experimentally obstructed colon. Colorectal Dis. 2011;13(7):e165-169.

14. Rutherfurd SM. Methodology for determining degree of hydrolysis of proteins in Hydrolysates: a review. J AOAC Int. 2010;93(5):1515-1522.

15. Knol MJ, VanderWeele TJ. Recommendations for presenting analyses of effect modification and interaction. Int J Epidemiol. 2012;41(2):514-520. 